\title{
Finite strain quadrilateral shell using least-squares fit of relative Lagrangian in-plane strains ${ }^{\text {th }}$
}

\author{
P. Areias ${ }^{\mathrm{a}, \mathrm{d}, *}$, T. Rabczuk ${ }^{\mathrm{b}}$, J.M. César de Sá ${ }^{\mathrm{c}}$, J.E. Garção ${ }^{\mathrm{a}, \mathrm{e}}$ \\ a Department of Physics, University of Évora, Colégio Luís António Verney, Rua Romão Ramalho, 59, 7002-554 Évora, Portugal \\ ${ }^{\mathrm{b}}$ Institute of Structural Mechanics, Bauhaus-University Weimar, Marienstraße 15, 99423 Weimar, Germany \\ ${ }^{c}$ Mechanical Engineering Department, Faculty of Engineering, University of Porto, Rua Dr. Roberto Frias, s/n, 4200-465 Porto, Portugal \\ d ICIST, Lisbon, Portugal \\ e IDMEC, Lisbon, Portugal
}

\section{A R T I C L E I N F O}

\section{Article history:}

Received 18 March 2014

Received in revised form

10 January 2015

Accepted 19 January 2015

\section{Keywords}

Finite strains

Shell elements

Pian-Sumihara stress modes

Finite strain plasticity

Least-square assumed strain

\begin{abstract}
A B S T R A C T
This work presents a finite strain quadrilateral element with least-squares assumed in-plane shear strains (in covariant/contravariant coordinates) and classical transverse shear assumed strains. It is an alternative to enhanced-assumed-strain (EAS) formulation and, in contrast to this, produces an element satisfying ab initio the Patch-test. No additional degrees-of-freedom are present, unlike EAS. Leastsquares fit allows the derivation of invariant finite strain elements which are both in-plane and out-ofplane shear-locking free and amenable to standardization in commercial codes. With that goal, we use automatically generated code produced by AceGen and Mathematica to obtain novel finite element formulations. The corresponding exact linearization of the internal forces was, until recently, a insurmountable task. We use the tangent modulus in the least-squares fit to ensure that stress modes are obtained from a five-parameter strain fitting. This reproduces exactly the in-plane bending modes. The discrete equations are obtained by establishing a four-field variational principle (a direct extension of the Hu-Washizu variational principle). The main achieved goal is coarse-mesh accuracy for distorted meshes, which is adequate for being used in crack propagation problems. In addition, as an alternative to spherical interpolation, a consistent director normalization is performed. Metric components are fully deduced and exact linearization of the shell element is performed. Full linear and nonlinear assessment of the element is performed, showing similar performance to more costly approaches, often on-par with the best available shell elements.
\end{abstract}

(c) 2015 Elsevier B.V. All rights reserved.

\section{Introduction}

Finite strain plasticity and fracture simulations with finite elements (cf. $[8,10]$ ) are peculiarly demanding with respect to numerical efficiency, Newton iteration robustness and mesh distortion insensitivity. This is relevant in the edge-based algorithms recently proposed [12] when applied to quadrilaterals. Many of the intricate element formulations, such as enhanced-assumed-strain, hybrid stress, discrete Kirchhoff (DK, cf. [14]), are suitable for smooth problems where the mesh distortion sensitivity is not a crucial factor and governing

${ }^{\sqrt{2}}$ Researcher ID: A-8849-2013, http://www.researcherid.com/rid/A-8849-2013 Web-of-Science search: areias, p*, Link to list of Journal papers: http:// home.uevora.pt/ pmaa/references.pdf.

* Corresponding author at: Department of Physics, University of Évora, Colégio Luís António Verney, Rua Romão Ramalho, 59, 7002-554 Évora, Portugal.

Tel.: + 35196 3496307; fax: + 351266745394.

E-mail addresses: pareias@civil.ist.utl.pt,pmaa@uevora.pt (P. Areias). equations do not contain discontinuities. In addition, costs associated with convergence difficulties and static condensation (specifically with EAS) can also be high. We take a different approach here: starting with a mixed 4-field functional (displacement field, director field, components of the local Cauchy-Green tensor and the corresponding stress-like Lagrange multipliers), we discretize the resulting Euler-Lagrange equations making use of suitable shape functions. A complete testing program is then performed. The set of obstacle problems for shells are the classical plate and shell benchmarks and extensions to finite strains. Testing elements in finite strains is also important since some instabilities have been found in the past (see [22] for a report with the Morley-based shell). Element technology for quadrilaterals is too vast to be accounted in a single article and many elements proposed in the last three decades vary only slightly in performance for the same number of degrees-of-freedom. Some important works must be mentioned. A milestone in the removal of transverse shear locking was achieved with the assumed natural strain (ANS) technique in 1984 and 1986 [24,36]. A decade earlier, 